\title{
Effects of Nicotine on Local Cerebral Glucose Utilization in the Rat
}

\author{
Edythe D. London, ${ }^{1}$ Robert J. Connolly, ${ }^{1}$ Margit Szikszay, ${ }^{1, a}$ James K. Wamsley, ${ }^{2}$ and Mauro Dam ${ }^{1, b}$ \\ ${ }^{1}$ Neuropharmacology Laboratory, Addiction Research Center, National Institute on Drug Abuse, Baltimore, Maryland \\ 21224, and 'Departments of Psychiatry, Pharmacology and Anatomy, University of Utah Medical Center, Salt Lake City, \\ Utah 84132
}

\begin{abstract}
We used the autoradlographic 2-deoxy-D-[1-14C]glucose $\left({ }^{14} \mathrm{C}-\right.$ DG) method of Sokoloff to identify brain areas with altered rates of local cerebral glucose utilization (LCGU) in vivo in response to peripheral /-nicotine administration $(0.1,0.3,1.0$, and $1.75 \mathrm{mg} / \mathrm{kg}, \mathrm{s.c}$.). Nicotine stimulated LCGU primarily in areas reported to contain nicotine binding sites, indicating that the sites are true receptors. Increases in LCGU of $100 \%$ or more over control were obtained in the medial habenula, fasciculus retroflexus, superior colliculus, and median eminence. Substantial stimulation (50-100\% increases) also was obtained in the cerebellar vermis, interpeduncular nucleus, and anteroventral and interanteromedial thalamic nuclei. Moderate increases $(20-50 \%$ ) were observed in the reticular nucleus of the medulla, paramedian lobule, nucleus of the spinal tract of the trigeminal nerve, presubiculum, subiculum, red nucleus, ventral tegmental area, substantia nigra, nucleus ambiguus, nucleus tractus solitarius, dorsal lateral geniculate nucleus, mammillothalamic tract, and fornix. The greatest stimulation in most affected areas was obtained with $0.3 \mathrm{mg} / \mathrm{kg}$ nicotine administered at $2 \mathrm{~min}$, but not longer, before ${ }^{14} \mathrm{C}$-DG. Effects of nicotine on LCGU were antagonized by mecamylamine. The findings indicate that the interaction of nicotine with specific binding sites is coupled to cerebral energy metabolism. The distribution of in vivo cerebral metabolic effects of nicotine implicates various brain regions in the behavioral and physiological effects of nicotine.
\end{abstract}

Nicotine produces many CNS effects, including memory facilitation, increases in spontaneous activity, convulsions, antinociception, suppression of irritability and appetite, and cardiovascular effects (Aceto and Martin, 1982; Larson and Silvette, $1968,1971,1975)$. It can serve as a reinforcer and is self-administered by laboratory animals and humans (Henningfield, 1984).

Interest in the cerebral loci where nicotine might act to produce these effects led to autoradiographic ligand-binding studies with ${ }^{3} \mathrm{H}$-nicotine (Clarke et al., 1984; London et al., 1985b). The studies demonstrated heterogeneous, specific binding in the rat brain, with dense labeling of the interpeduncular nucleus,

\footnotetext{
Received Jan. 6, 1988; revised Feb. 18, 1988; accepted Mar. 7, 1988.

Correspondence should be addressed to Dr. E. D. London, NIDA Addiction Research Center, P.O. Box 5180, Baltimore, MD 21224.

${ }^{a}$ On leave from Department of Physiology, University Medical School, Dom Ter 10, H-6720, Szeged, Hungary.

b Present address: Universita Degli Studi Padova, Instituto di Clinica della Malattie Nervose e Mentali, Via Guistiniani 5, 35100 Padova, Italy.

Copyright (C) 1988 Society for Neuroscience $0270-6474 / 88 / 103920-09 \$ 02.00 / 0$
}

medial habenula, thalamic nuclei, sensory areas, and the cerebral cortex. Specific ${ }^{3} \mathrm{H}$-nicotine binding sites showed a high concordance with ${ }^{3} \mathrm{H}-\Lambda \mathrm{Ch}$ binding, suggesting that ${ }^{3} \mathrm{H}$-nicotine binds to nicotinic ACh receptors (Clarke et al., 1985).

The purpose of the present study was to delineate the in vivo loci of nicotine effects in the brain and to compare the distribution with locations of specific ${ }^{3} \mathrm{H}$-nicotine binding sites. We therefore used the autoradiographic 2-deoxy-D-1-[ $\left.{ }^{4} \mathrm{C}\right]$ glucose $\left({ }^{14} \mathrm{C}-\mathrm{DG}\right)$ method to measure local cerebral glucose utilization (LCGU) (Sokoloff et al., 1977). Rates of LCGU have provided an index of local cerebral function under a wide variety of conditions (Sokoloff, 1977), including brain stimulation (Esposito et al., 1984) and selective lesions (London et al., 1984; Ito et al., 1985). The ${ }^{14} \mathrm{C}-\mathrm{DG}$ method also has been used to map the in vivo distributions of action of various drugs (McCulloch, 1982).

\section{Materials and Methods}

Animals and their preparation. Three-month-old male Fischer-344 rats were obtained from Charles River Breeding Laboratories (Wilmington, MA). They were housed, 2 per cage, in a temperature-controlled vivarium $\left(23 \pm 1{ }^{\circ} \mathrm{C}\right)$ with alternating light-dark cycles (lights on, 0600 ; lights off, 1800) and were allowed to acclimate to this environment for $2-4$ weeks before experimentation. Food and water were available ad libitum until the evening prior to each ${ }^{14} \mathrm{C}-\mathrm{DG}$ experiment.

Rats had free access to water but were deprived of food for about 15 $\mathrm{hr}$ before surgery. Under halothane anesthesia, they were prepared with indwelling catheters in the left femoral artery and vein. They were partially immobilized in plaster casts that covered their hindquarters and were allowed to recover from anesthesia for $3 \mathrm{hr}$ before drug treatments in a temperature-controlled, sound-insulated wooden box (London et al., 1981).

Blood pressure was monitored by connecting the arterial catheter to a strain gauge transducer (Statham Instruments Co., Hatorey, PR), and the output was displayed on a chart recorder (Gould Recorder 2200, Gould Inc., Cleveland, $\mathrm{OH}$ ).

Behavioral effects of nicotine were assessed in the partially immobilized rats at three time intervals after nicotine injection: $0-10 \mathrm{~min}$, 10-25 min, and 25-90 min. The presence of the following effects was recorded: Straub tail, increased restlessness (manifested as head swinging, forelimb grasping, paddling, tail wagging, and struggling against the restraint of the cast), tremor, and convulsive movements, consisting of facial fasciculations and forelimb clonus.

Drug treatments. l-Nicotine bitartrate (BDH Chemicals Ltd., Poole, England) was dissolved in $0.9 \% \mathrm{NaCl}$, and the $\mathrm{pH}$ of the solution was adjusted to 7.0 with $1 \mathrm{~N} \mathrm{NaOH}$. Animals were given s.c. injections of nicotine $(1 \mathrm{ml} / \mathrm{kg})$ or an equal volume of $0.9 \% \mathrm{NaCl}$. Nicotine effects were tested at doses of $0.1,0.3,1.0$, and $1.75 \mathrm{mg} / \mathrm{kg}$. The treatments were administered at $2,15,30,60$, or $120 \mathrm{~min}$ before the radiotracer.

In one group of rats, $2.5 \mathrm{mg} / \mathrm{kg}$ mecamylamine $\mathrm{HCl}$ (Merck, Rahway, $\mathrm{NJ}$ ) was injected s.c. $20 \mathrm{~min}$ before $1.0 \mathrm{mg} / \mathrm{kg}$ nicotine. Hexamethonium bromide (Sigma, St. Louis), $4 \mathrm{mg} / \mathrm{kg}$, was injected i.p. $10 \mathrm{~min}$ before $1.75 \mathrm{mg} / \mathrm{kg}$ nicotine. Drug dosages were calculated as the free bases.

Determination of $L C G U$. The radiotracer $\left({ }^{14} \mathrm{C}-\mathrm{DG}\right.$; specific activity, 
55-57 mCi/mmol; Amersham Corp., Arlington Heights, IL) was infused intravenously over about $6 \mathrm{sec}$, at a dose of $125 \mu \mathrm{Ci} / \mathrm{kg}$ body weight. Blood samples were collected, and plasma was prepared and assayed for ${ }^{14} \mathrm{C}$-DG and glucose concentrations as described previously (Sokoloff et al., 1977). Rats were killed $45 \mathrm{~min}$ after the ${ }^{14} \mathrm{C}-\mathrm{DG}$ injection by an intravenous overdose of sodium pentobarbital $(60 \mathrm{mg}$ in $1 \mathrm{ml}$, Abbott Laboratories, North Chicago, IL).

Brains were removed and frozen in 2-methylbutane cooled to $-60^{\circ} \mathrm{C}$ by dry ice. Frozen $20 \mu \mathrm{m}$ brain sections were cut in a cryostat, dried quickly, and apposed to Kodak SB-5 film along with calibrated ${ }^{14} \mathrm{C}-$ methyl methacrylate standards (Amersham) for $7 \mathrm{~d}$. Optical densities were determined from the resultant autoradiograms in areas corresponding to 82 brain regions using a Leitz Orthoplan microscope equipped with a MPV microdensitometer and microprocessor (model 560/DADS, E. Leitz, Rockleigh, NJ). Anatomical areas were defined by comparing autoradiograms with a stereotaxic atlas (Paxinos and Watson, 1982). Values of LCGU were calculated from brain and plasma radioactivities and plasma glucose concentrations, using an operational equation and the rate and lumped constants for the bidirectional transport of ${ }^{14} \mathrm{C}$-DG between the brain and plasma and ${ }^{14} \mathrm{C}-\mathrm{DG}$ phosphorylation in brain as given by Sokoloff et al. (1977).

Statistical analysis. Effects of drug treatments on mean arterial blood pressure were assessed using a 2-way analysis of covariance, with the drug treatments (saline; $0.1,0.3$, and $1.0 \mathrm{mg} / \mathrm{kg}$ nicotine) and sampling times (baseline, 6, 12, and $30 \mathrm{~min}$ after ${ }^{14} \mathrm{C}-\mathrm{DG}$ ) as main effects and time as a repeated measure. The drug treatments and times were taken as the variables and baseline parameter values as the covariates. Individual means were compared using Tukey's $\omega$ procedure. In a separate analysis, the effect of $1.0 \mathrm{mg} / \mathrm{kg}$ nicotine on mean arterial blood pressure was determined in the presence and absence of mecamylamine. As the effect of time had already been tested in the aforementioned analysis of covariance, a separate 1-way analysis of variance was performed on the data from each time point (baseline, 6, 12, and $30 \mathrm{~min}$ after ${ }^{14} \mathrm{C}-\mathrm{DG}$ ), and Bonferroni $t$ test was used to compare the means.

The significance of drug treatments on LCGU (varying doses) was determined by 1-way analysis of variance. Dunnett's test was performed on data from individual brain regions where significant $F$ values were found, in order to evaluate group differences. Time course data from the 20 brain regions in which $1.0 \mathrm{mg} / \mathrm{kg}$ nicotine significantly affected LCGU in the aforementioned dose-response study also were subjected to Dunnett's test. Student's $t$ test was used to compare the mean LCGU values in rats treated with mecamylamine + nicotine to those in control rats. The criterion for all significance statements was $p \leq 0.05$.

\section{Results}

\section{Hypertensive effect}

Analysis of the data on mean arterial blood pressure yielded significant $F$ values for each main effect (dose, $F(3,12)=11.8$; time, $F(2,26)=14.2)$ and for the interaction $(F(6,26)=4.52)$. All doses of nicotine elevated mean arterial blood pressure as compared with saline control at all observation times $(6,12$, and 30 min after ${ }^{14} \mathrm{C}-\mathrm{DG}$ injection; see Fig. 1 for values in salinetreated rats). The highest blood pressure occurred at $6 \mathrm{~min}$ in rats treatcd with $1.0 \mathrm{mg} / \mathrm{kg}$ nicotine $(144 \pm 2.7 \mathrm{~mm} \mathrm{IIg} \mathrm{vs} 110$ $\pm 6.3 \mathrm{~mm} \mathrm{Hg}$ in controls). At this time, rats in the $1.0 \mathrm{mg} / \mathrm{kg}$ group showed a higher pressure than animals treated with lower doses of nicotine $(0.1 \mathrm{mg} / \mathrm{kg}, 119 \pm 4.0 \mathrm{~mm} \mathrm{Hg} ; 0.3 \mathrm{mg} / \mathrm{kg}$, $124 \pm 3 \mathrm{~mm} \mathrm{Hg}$ ). The difference between the $1.0 \mathrm{mg} / \mathrm{kg}$ group and the 0.1 and $0.3 \mathrm{mg} / \mathrm{kg}$ groups was not seen at the other observation points.

Prior treatment of rats with mecamylamine prevented nicotine-induced hypertension at every time point assayed (Fig. 1). In addition, 3 rats pretreated with hexamethonium before 1.75 $\mathrm{mg} / \mathrm{kg}$ nicotine showed blood pressure values similar to those in saline-treated rats (data not shown).

\section{Behavioral effects}

Nicotine produced dose- and time-dependent effects on motor and Straub-tail responses (Fig. 2). The Straub-tail response was

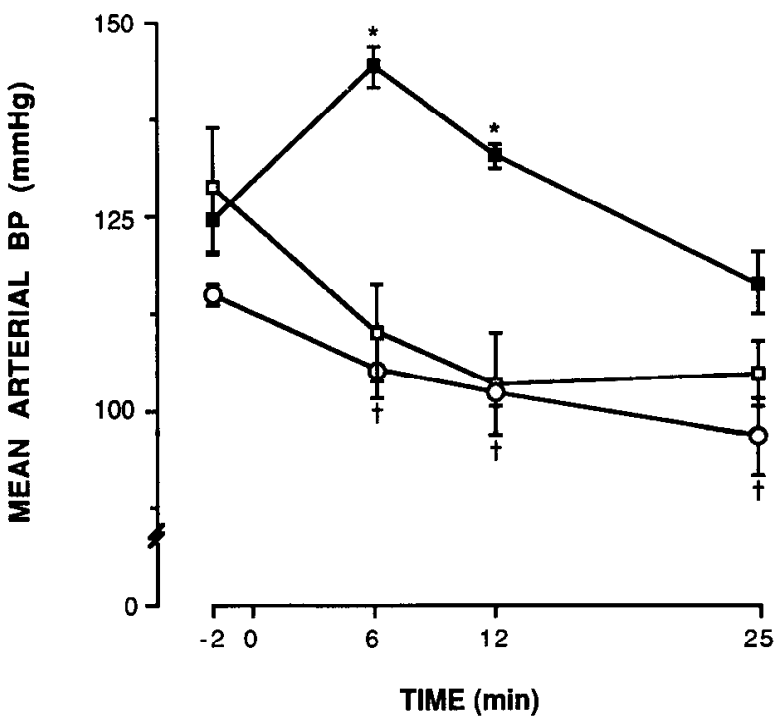

Figure 1. Antagonism of nicotine-induced hypertension by mecamylamine. Mean arterial blood pressure is shown at various times relative to the injection of ${ }^{14} \mathrm{C}-\mathrm{DG}$. Animals received $0.9 \% \mathrm{NaCl}(1 \mathrm{ml}$

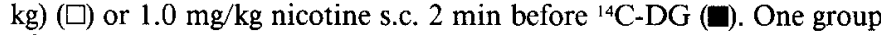
of rats received $2.5 \mathrm{mg} / \mathrm{kg}$ mecamylamine $\mathrm{HCl}$ s.c. $20 \mathrm{~min}$ before 1.0 Ing $/ \mathrm{kg}$ nicotine (O). Data shown are the means \pm SEM for 3-5 rats per group. * Significant difference from saline control $(p \leq 0.05$ by Bonferroni $t$ test); $\dagger$ significant difference from nicotine alone $(p \leq 0.05$ by Bonferroni $t$ test).

seen after 0.3 or 1.0 , but not $0.1 \mathrm{mg} / \mathrm{kg}$ nicotine. The response dissipated within $12 \mathrm{~min}$ after the $0.3 \mathrm{mg} / \mathrm{kg}$ nicotine injection (10 min after the ${ }^{14} \mathrm{C}$-DG injection) but lasted up to $90 \mathrm{~min}$ after the injection of $1.0 \mathrm{mg} / \mathrm{kg}$ nicotine. Signs of increased motor activity or restlessness and tremor were observed after $0.1,0.3$, and $1.0 \mathrm{mg} / \mathrm{kg}$ nicotine at the earliest measurement time. Increased restlessness also was apparent at 10-25 min but not at later times after the $0.3 \mathrm{mg} / \mathrm{kg}$ dose. The response persisted into the last observation period (25-90 min after the nicotine injection) in rats treated with $1.0 \mathrm{mg} / \mathrm{kg}$ nicotine. 'I remor was seen at the earliest observation time after all nicotine doses, but persisted only after $1.0 \mathrm{mg} / \mathrm{kg}$. Convulsive movements were seen only after $1.0 \mathrm{mg} / \mathrm{kg}$ nicotine, and lasted into the 10-25 min observation period.

Because the behavioral testing was carried out using rats that were partially immobilized for the LCGU experiments, it seemed that immobilization stress could influence the behaviors which were scored. Neither saline injection nor the partial immobilization induced any of the aforementioned behavior observed, although an interaction of immobilization stress with nicotine treatment cannot be ruled out.

\section{Effects on $L C G U$}

Nicotine, injected $2 \mathrm{~min}$ before ${ }^{14} \mathrm{C}$-DG, produced selective, dose-dependent LCGU increases in 21 of the brain regions analyzed (Table 1). No LCGU decrements were observed.

Only 3 regions showed significantly elevated LCGU after treatment with $0.1 \mathrm{mg} / \mathrm{kg}$ nicotine. These areas were the subiculum, superficial gray layers of the superior colliculus, and the nucleus ambiguus, in which LCGU was elevated by $27 \%, 60 \%$, and $33 \%$ over control, respectively.

Higher doses of nicotine increased LCGU significantly, by $28 \%-161 \%$ over control. In most areas where nicotine increased LCGU, the maximal effect was obtained with $0.3 \mathrm{mg} / \mathrm{kg}$ nico- 
Table 1. Effects of nicotine on local cerebral glucose utilization

\begin{tabular}{|c|c|c|c|c|}
\hline \multirow[b]{2}{*}{ Tissue } & \multirow[b]{2}{*}{$\begin{array}{l}\text { Saline control } \\
(n=7)\end{array}$} & \multicolumn{3}{|l|}{ Nicotine } \\
\hline & & $\begin{array}{l}0.1 \mathrm{mg} / \mathrm{kg} \\
(n=5)\end{array}$ & $\begin{array}{l}0.3 \mathrm{mg} / \mathrm{kg} \\
(n=4)\end{array}$ & $\begin{array}{l}1.0 \mathrm{mg} / \mathrm{kg} \\
(n=6)\end{array}$ \\
\hline Cuneate nucleus (n.) & $57 \pm 4.1$ & $66 \pm 4.6$ & $79 \pm 9.5$ & $76 \pm 6.5$ \\
\hline Reticular n. of medulla, dorsal & $53+1.3$ & $62+3.1$ & $68+5.8^{*}$ & $59 \pm 3.2$ \\
\hline Cerebellar vermis $^{a}$ & $63 \pm 4.1$ & $73 \pm 5.2$ & $105 \pm 7.7^{*}$ & $108 \pm 13.5^{*}$ \\
\hline Paramedian lobule & $49 \pm 2.6$ & $52 \pm 1.5$ & $66 \pm 3.6^{*}$ & $58 \pm 3.2$ \\
\hline Inferior olive & $69 \pm 3.0$ & $73 \pm 4.2$ & $83 \pm 2.7$ & $79 \pm 4.0$ \\
\hline Medial vestibular $n$. & $114 \pm 9.2$ & $135 \pm 4.6$ & $137 \pm 10.6$ & $150 \pm 9.7$ \\
\hline Interpositus cerebellar $n$. & $101 \pm 8.6$ & $105 \pm 6.4$ & $121 \pm 12.5$ & $116 \pm 6.2$ \\
\hline Dorsal cochlear $\mathrm{n}$. & $132 \pm 16.0$ & $116 \pm 5.6$ & $136 \pm 12.5$ & $107 \pm 5.3$ \\
\hline Locus ceruleus & $67 \pm 3.7$ & $71 \pm 2.9$ & $73 \pm 3.3$ & $76 \pm 5.5$ \\
\hline Dorsal tegmental $\mathrm{n}$. & $94 \pm 7.3$ & $94 \pm 5.8$ & $95 \pm 5.9$ & $97 \pm 7.8$ \\
\hline Central gray & $63 \pm 4.1$ & $69 \pm 4.5$ & $74 \pm 9.1$ & $71 \pm 3.6$ \\
\hline Nucleus of the spinal tract of trigeminal nerve, dorsal & $52 \pm 1.6$ & $62 \pm 2.6$ & $71 \pm 5.7^{*}$ & $62 \pm 4.0$ \\
\hline Pontine reticular n., caudal part & $56 \pm 3.6$ & $59 \pm 3.0$ & $66 \pm 3.5$ & $60 \pm 4.2$ \\
\hline Median raphe $\mathrm{n}$. & $101 \pm 7.4$ & $110 \pm 5.0$ & $116 \pm 8.2$ & $114 \pm 5.5$ \\
\hline Dorsal raphe $\mathrm{n}$. & $92 \pm 4.1$ & $98 \pm 6.2$ & $110 \pm 10.1$ & $100 \pm 5.0$ \\
\hline Ventral $\mathrm{n}$. of lateral lemniscus & $118 \perp 10.3$ & $118 \pm 6.2$ & $124 \pm 8.5$ & $98 \pm 7.0$ \\
\hline Inferior colliculus & $170 \pm 13.4$ & $179 \pm 11.1$ & $180 \pm 15.8$ & $150 \pm 8.4$ \\
\hline Pontine nuclei & $62 \pm 3.6$ & $63 \pm 1.9$ & $77 \pm 6.9$ & $67 \pm 2.7$ \\
\hline Presubiculum & $100 \pm 7.2$ & $119 \pm 3.7$ & $133 \pm 12.5^{*}$ & $143 \pm 10.7^{*}$ \\
\hline Subiculum & $84 \pm 4.9$ & $107 \pm 3.4^{*}$ & $114 \pm 8.7^{*}$ & $110 \pm 7.2^{*}$ \\
\hline Superficial gray, superior colliculus & $78 \pm 5.2$ & $125 \pm 12.5^{*}$ & $165 \pm 13.1^{*}$ & $149 \pm 5.6^{*}$ \\
\hline Red $n$. & $76 \pm 6.2$ & $86 \pm 2.7$ & $103 \pm 7.2^{*}$ & $97 \pm 6.1^{*}$ \\
\hline Deep mesencephalic n. & $67 \pm 4.4$ & $73 \pm 3.3$ & $83 \pm 5.5$ & $75 \pm 5.9$ \\
\hline Ventral tegmental $\mathrm{n}$. & $94 \pm 8.7$ & $108 \pm 5.2$ & $115 \pm 9.8$ & $114 \pm 4.0$ \\
\hline Ventral tegmental area & $66 \pm 4.2$ & $76 \pm 2.9$ & $96 \pm 9.9 *$ & $90 \pm 6.1^{*}$ \\
\hline Interpeduncular n., central & $115 \pm 7.6$ & $126 \pm 5.3$ & $174 \pm 13.1^{*}$ & $169 \pm 8.3^{*}$ \\
\hline Medial geniculate n., dorsal & $111 \pm 7.6$ & $131 \pm 3.4$ & $133 \pm 7.5$ & $119 \pm 8.0$ \\
\hline Lateral geniculate n., dorsal & $83 \pm 5.9$ & $96 \pm 3.7$ & $121 \pm 8.1^{*}$ & $114 \pm 10.6^{*}$ \\
\hline Anterior pretectal area & $105 \pm 9.0$ & $120 \pm 9.7$ & $128 \pm 9.7$ & $130 \pm 8.8$ \\
\hline Substantia nigra pars compacta & $72 \pm 4.6$ & $76 \pm 4.6$ & $101 \pm 8.5^{*}$ & $98 \pm 10.2^{*}$ \\
\hline Medial mammillary $n$. & $118 \pm 9.1$ & $114 \pm 6.6$ & $142 \pm 7.7$ & $143 \pm 13.2$ \\
\hline Dorsal hippocampus, CAl & $61 \pm 5.6$ & $58 \pm 3.0$ & $63 \pm 4.6$ & $53 \pm 5.6$ \\
\hline Dorsal hippocampus, CA3 & $72 \pm 5.5$ & $71 \pm 3.8$ & $72 \pm 7.5$ & $61 \pm 6.1$ \\
\hline Dentate gyrus & $57 \pm 3.4$ & $58 \pm 3.7$ & $65 \pm 4.4$ & $49 \pm 3.9$ \\
\hline Medial habenular n. & $91 \pm 11.0$ & $90 \pm 2.9$ & $118 \pm 8.9$ & $192 \pm 18.3^{*}$ \\
\hline Lateral habenular $\mathrm{n}$. & $115 \pm 9.3$ & $131 \pm 6.4$ & $138 \pm 9.0$ & $114 \pm 8.0$ \\
\hline Subthalamic $\mathrm{n}$ & $91 \pm 7.5$ & $97 \pm 5.2$ & $103 \pm 10.6$ & $98 \pm 10.2$ \\
\hline \multicolumn{5}{|l|}{ Thalamic nuclei } \\
\hline Ventromedial & $49 \pm 4.5$ & $45 \pm 3.1$ & $63 \pm 5.8$ & $50 \pm 3.6$ \\
\hline Posterior & $65 \pm 4.8$ & $67 \pm 3.8$ & $78 \pm 5.3$ & $77 \pm 4.5$ \\
\hline Lateral posterior & $95+11.5$ & $126+5.1$ & $122+5.7$ & $120+9.7$ \\
\hline Ventroposterior & $94 \pm 5.8$ & $102 \pm 9.1$ & $113 \pm 5.6$ & $95 \pm 5.4$ \\
\hline Ventrolateral & $111 \pm 11.2$ & $115 \pm 6.6$ & $131 \pm 11.0$ & $133 \pm 10.6$ \\
\hline Mediodorsal & $115 \pm 10.7$ & $133 \pm 8.3$ & $142 \pm 11.8$ & $139 \pm 11.1$ \\
\hline Paratenial & $103 \pm 11.6$ & $113 \pm 11.7$ & $102 \pm 3.6$ & $93 \pm 8.8$ \\
\hline Anteroventral & $117 \pm 7.2$ & $146 \pm 9.0$ & $186 \pm 7.0^{*}$ & $208 \pm 18.9^{*}$ \\
\hline Interanteromedial & $121 \pm 5.9$ & $155 \pm 9.7$ & $178 \pm 10.1^{*}$ & $181 \pm 18.9^{*}$ \\
\hline Reticular & $94 \pm 8.9$ & $113 \pm 9.3$ & $122 \pm 8.1$ & $120 \pm 12.0$ \\
\hline Gelatinosus & $117 \pm 10.2$ & $125 \pm 9.2$ & $152 \pm 13.0$ & $141 \pm 14.5$ \\
\hline \multicolumn{5}{|l|}{ Hypothalamus } \\
\hline Supraoptic n. & $120 \pm 9.5$ & $107 \pm 8.9$ & $123 \pm 9.2$ & $103 \pm 6.9$ \\
\hline Dorsomedial n. & $56 \pm 5.4$ & $61 \pm 4.4$ & $74 \pm 7.9$ & $66 \pm 7.6$ \\
\hline Median eminence & $49 \pm 3.4$ & $63 \pm 5.0$ & $128 \pm 21.3^{*}$ & $96 \pm 3.9^{*}$ \\
\hline Anterior area & $53 \pm 4.6$ & $87 \pm 8.6$ & $91 \pm 13.6$ & $73 \pm 7.3$ \\
\hline Suprachiasmatic n. & $83 \pm 8.3$ & $87 \pm 8.6$ & $91 \pm 13.6$ & $73 \perp 7.3$ \\
\hline Basolateral amygdaloid n. & $90 \pm 5.7$ & $93 \pm 7.3$ & $111 \pm 8.1$ & $108 \pm 7.2$ \\
\hline
\end{tabular}


Table 1. Continued

\begin{tabular}{|c|c|c|c|c|}
\hline \multirow[b]{2}{*}{ Tissue } & \multirow[b]{2}{*}{$\begin{array}{l}\text { Saline control } \\
(n=7)\end{array}$} & \multicolumn{3}{|l|}{ Nicotine } \\
\hline & & $\begin{array}{l}0.1 \mathrm{mg} / \mathrm{kg} \\
(n=5)\end{array}$ & $\begin{array}{l}0.3 \mathrm{mg} / \mathrm{kg} \\
(n=4)\end{array}$ & $\begin{array}{l}1.0 \mathrm{mg} / \mathrm{kg} \\
(n=6)\end{array}$ \\
\hline Globus pallidus & $55 \pm 3.6$ & $55 \pm 7.6$ & $60 \pm 3.1$ & $53 \pm 5.5$ \\
\hline Caudate putamen & $91 \pm 10.8$ & $94 \pm 5.0$ & $106 \pm 6.9$ & $107 \pm 8.9$ \\
\hline Medial septal $n$. & $83 \pm 8.1$ & $77 \pm 5.5$ & $86 \pm 9.3$ & $89 \pm 7.4$ \\
\hline N. vertical limb of diagonal band & $78 \pm 6.3$ & $79 \pm 5.9$ & $90 \pm 10.4$ & $82 \pm 7.6$ \\
\hline Lateral septal $\mathbf{n}$. & $53 \pm 3.2$ & $56 \pm 4.3$ & $61 \pm 2.2$ & $58 \pm 4.1$ \\
\hline Septofimbrial $\mathrm{n}$. & $71 \pm 3.0$ & $78 \pm 7.4$ & $92 \pm 8.6$ & $81 \pm 5.3$ \\
\hline Accumbens $\mathrm{n}$. & $94 \pm 6.0$ & $90 \pm 7.7$ & $90 \pm 14.3$ & $100 \pm 7.6$ \\
\hline Olfactory tubercle & $125 \pm 10.7$ & $108 \pm 7.8$ & $146 \pm 23.4$ & $129 \pm 8.3$ \\
\hline Ambiguus $\mathrm{n}$. & $58 \pm 3.5$ & $77 \pm 5.9^{*}$ & $81 \perp 7.6^{*}$ & $70 \pm 4.4$ \\
\hline N. solitary tract & $59 \pm 2.2$ & $69 \pm 4.0$ & $73 \pm 3.6$ & $76 \pm 4.8^{*}$ \\
\hline \multicolumn{5}{|l|}{ Cerebral cortex ${ }^{b}$} \\
\hline Striate, area 17 & $110 \pm 5.7$ & $105 \pm 7.6$ & $114 \pm 11.2$ & $104 \pm 5.5$ \\
\hline Retrosplenial, superficial layers & $113 \pm 7.8$ & $119 \pm 6.3$ & $132 \pm 7.2$ & $125 \pm 5.4$ \\
\hline Retrosplenial, deep layer & $102 \pm 9.0$ & $112 \pm 9.3$ & $120 \pm 8.5$ & $107 \pm 5.0$ \\
\hline Temporal, auditory area & $131 \pm 8.1$ & $145 \pm 9.8$ & $170 \pm 16.5$ & $135 \pm 8.9$ \\
\hline Frontoparietal, motor area & $105 \pm 7.9$ & $94 \pm 9.7$ & $121 \pm 8.0$ & $101 \pm 6.0$ \\
\hline Anterior cingulate, superficial layers & $110 \pm 4.3$ & $100 \pm 10.2$ & $132 \pm 13.0$ & $119 \pm 13.8$ \\
\hline Anterior cingulate, deep layers & $111 \pm 7.9$ & $112 \pm 10.4$ & $123 \pm 11.3$ & $111 \pm 10.7$ \\
\hline Frontal & $103 \pm 8.4$ & $91 \pm 7.8$ & $114 \pm 7.6$ & $97 \pm 6.2$ \\
\hline Primary olfactory & $111 \pm 10.6$ & $97 \pm 6.3$ & $124 \pm 15.4$ & $111 \pm 7.4$ \\
\hline \multicolumn{5}{|l|}{ White matter areas } \\
\hline Fasciculus retroflexus & $94 \pm 6.9$ & $107 \pm 1.6$ & $132 \pm 7.7^{*}$ & $202 \pm 15.1^{*}$ \\
\hline Mammillothalamic tract & $77 \pm 3.4$ & $84 \pm 3.5$ & $100 \pm 10.1^{*}$ & $104 \pm 4.8^{*}$ \\
\hline Fornix & $64 \pm 3.5$ & $72 \pm 3.6$ & $89 \pm 12.2^{*}$ & $91 \pm 4.5^{*}$ \\
\hline Corpus callosum & $36 \pm 2.8$ & $39 \pm 4.1$ & $42 \pm 3.8$ & $33 \pm 3.3$ \\
\hline Medial forebrain bundle & $64 \pm 4.5$ & $70 \pm 5.8$ & $83 \pm 8.0$ & $65 \pm 5.9$ \\
\hline Stria medullaris of thalamus & $51 \pm 2.7$ & $51 \pm 4.4$ & $54 \pm 1.8$ & $47 \pm 5.6$ \\
\hline
\end{tabular}

l-Nicotine bitartrate was injected subcutaneously in $1 \mathrm{ml} / \mathrm{kg}$ body weight of $0.9 \% \mathrm{NaCl}$ (wt/vol) at $2 \mathrm{~min}$ before ${ }^{14} \mathrm{C}$-DG. Rats given injections with the same volume of $0.9 \% \mathrm{NaCl}$ served as a control group. Glucose utilization was determined as previously described (Sokoloff et al., 1977). For each brain region, at least 6 densitometric readings were taken from no less than 3 tissue sections. Doses of nicotine are expressed as the free base. The values for $L C G U(\mu \mathrm{mol} / 100 \mathrm{gm}$ tissue/min) are expressed as means \pm SEM for the number of rats indicated in parentheses. Asterisks indicate significant difference from control $(p<0.05$ by 1 -way ANOVA and Dunnett's test).

${ }^{\alpha}$ Densitometric readings were taken from the granular layer of the cerebellar vermis. In nicotine-treated rats, hypermetabolic spots were noted in this area (Fig. 2); readings were taken from the regions adjacent to the spots.

${ }^{b}$ For all neocortical areas, data shown are for layer 4. Readings also were taken from layers $1-3$ and 5 of each area; however, no significant treatment effects were observed (data not shown).

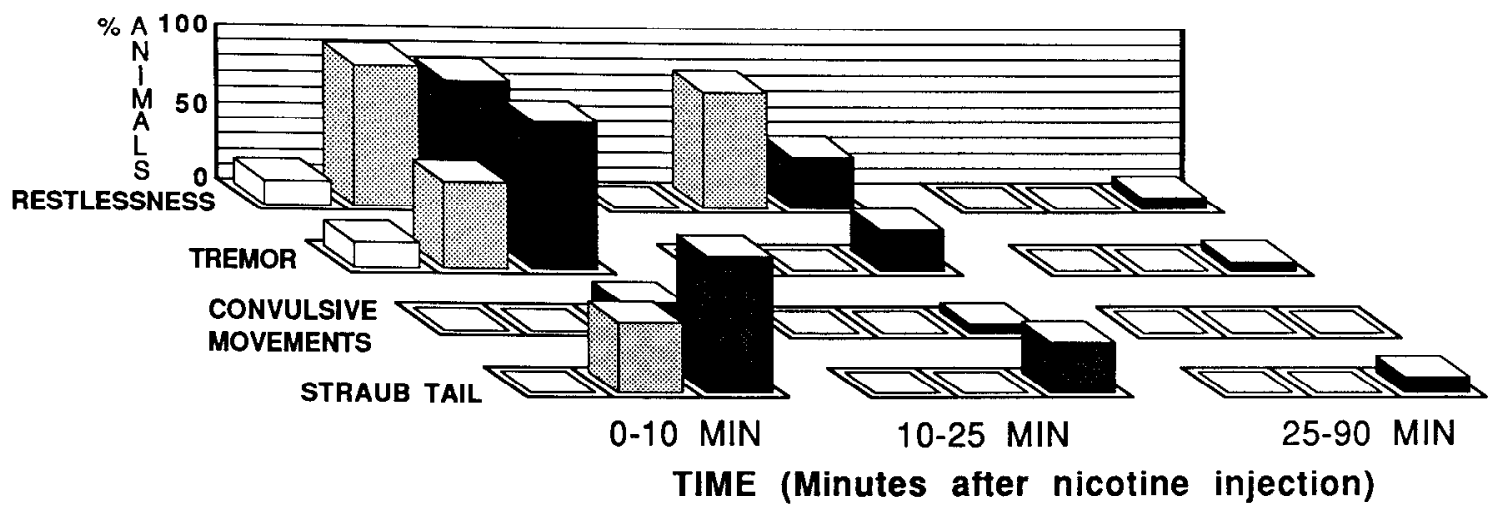

Figure 2. Behavioral effects of nicotine in partially immobilized rats. Nicotine $(0.1, \square ; 0.3, \square ;$ or $1.0 \mathrm{mg} / \mathrm{kg}, \mathbf{\square}$, s.c.; $n=5,7$, and 22 , respectively) was injected at 2,15 , or 60 min before ${ }^{14} \mathrm{C}-\mathrm{DG}$, and the rats were observed from the time of the nicotine injection until they were killed at 45 min after the injection of ${ }^{14} \mathrm{C}-\mathrm{DG}$. Rats that received 0.1 or $0.3 \mathrm{mg} / \mathrm{kg}$ nicotine were given ${ }^{14} \mathrm{C}-\mathrm{DG} 2 \mathrm{~min}$ later, whereas those that received $1 \mathrm{mg} / \mathrm{kg}$ nicotine were given injections of the radiotracer at 2, 15, or 60 min later. Restlessness or increased motor activity was seen as head swinging, forelimb grasping, paddling, tail wagging, and struggling. The observed tremor was apparent in the face and rest of head, forelimbs, and tail, and was accompanied by teeth chattering. Convulsive movements included facial fasciculations and forelimb clonus. 
Table 2. Time course of nicotine's effects on local cerebral glucose utilization

\begin{tabular}{|c|c|c|c|c|}
\hline \multirow[b]{2}{*}{ Tissue } & \multicolumn{4}{|l|}{ Nicotine } \\
\hline & $\begin{array}{l}2 \min \\
(n=6)\end{array}$ & $\begin{array}{l}15 \mathrm{~min} \\
(n=5) \\
\end{array}$ & $\begin{array}{l}60 \text { min } \\
(n=5) \\
\end{array}$ & $\begin{array}{l}120 \min \\
(n=4)\end{array}$ \\
\hline Cerebellar vermis ${ }^{a}$ & $171 \pm 21$ & $114 \pm 10$ & $122 \pm 5.0$ & $122 \pm 7.0$ \\
\hline Presubiculum & $143 \pm 11^{*}$ & $105 \pm 6.2$ & $111 \pm 13$ & $118 \pm 6.5$ \\
\hline Subiculum & $131 \pm 9.0^{*}$ & $100 \pm 5.0$ & $108 \pm 8.0$ & $123 \pm 7.0$ \\
\hline Superficial gray, superior colliculus & $191 \pm 7.0^{*}$ & $171 \pm 8.0^{*}$ & $173 \pm 19^{*}$ & $179 \pm 11^{*}$ \\
\hline Ventral tegmental area & $136 \pm 9.2^{*}$ & $114 \pm 5.0$ & $118 \pm 9.10$ & $129 \pm 9.7$ \\
\hline Interpeduncular n., central & $147 \pm 7.2^{*}$ & $110 \pm 3.9$ & $109 \pm 11$ & $119 \pm 9.9$ \\
\hline Dorsal lateral geniculate $n$. & $137 \pm 13^{*}$ & $114 \pm 8.4$ & $122 \pm 12$ & $131 \pm 10$ \\
\hline Substantia nigra pars compacta & $136 \pm 14^{*}$ & $110 \pm 17$ & $107 \pm 6.4$ & $117 \pm 14$ \\
\hline Medial habenular $\mathrm{n}$. & $211 \pm 20^{*}$ & $169 \pm 14^{*}$ & $143 \pm 6.0$ & $106 \pm 8.6$ \\
\hline Anteroventral thalamic $\mathrm{n}$. & $178 \pm 16^{*}$ & $122 \pm 7.5$ & $133 \pm 17$ & $121 \pm 9.0$ \\
\hline N. solitary tract & $129 \pm 6.8^{*}$ & $107 \pm 9.8$ & $98 \pm 12$ & $110 \pm 3.7$ \\
\hline Fasciculus retroflexus & $215 \pm 16^{*}$ & $132 \pm 7.0$ & $132 \pm 12$ & $123 \pm 9.9$ \\
\hline Mammillothalamic tract & $135 \pm 6.2^{*}$ & $97.4 \pm 4.5$ & $109 \pm 10$ & $105 \pm 6.2$ \\
\hline Fornix & $142 \pm 7.0^{*}$ & $103 \pm 5.9$ & $108 \pm 14$ & $116 \pm 5.5$ \\
\hline
\end{tabular}

l-Nicotine bitartrate $(1.0 \mathrm{mg} / \mathrm{kg}$, free base) was injected subcutaneously in $1 \mathrm{ml} / \mathrm{kg}$ body weight of $0.9 \% \mathrm{NaCl}$ (wt/vol) at various times before ${ }^{14} \mathrm{C}-\mathrm{DG}$. Glucose utilization $(\mu \mathrm{mol} / 100 \mathrm{gm}$ tissue/min) was determined as previously described (Sokoloff et al., 1977). For each brain region, at least 6 densitometric readings were taken from no less than 3 tissue sections. Effects on LCGU, expressed as percentages of the controls, are shown for selected brain regions in which LCGU was significantly different $2 \mathrm{~min}$ after $1 \mathrm{mg} / \mathrm{kg}$ nicotine than in corresponding control rats, which received injections containing the same volume of $0.9 \% \mathrm{NaCl}(p \leq 0.05$; see Table 1 for control values). Asterisks indicate significant difference from control ( $p \leq 0.05$ by Dunnett's test).

${ }^{a}$ Densitometric readings were taken from the granular layer of the cerebellar vermis. In nicotine-treated rats, hypermetabolic spots were noted in this area (Fig. 3); readings were taken from the regions adjacent to the spots.

tine, with no further greater effect of $1.0 \mathrm{mg} / \mathrm{kg}$. Examples of this relation were obtained in the following brain areas, where effects on LCGU were statistically significant after treatment with 0.3 as well as $1.0 \mathrm{mg} / \mathrm{kg}$ nicotine (numbers in parentheses indicate mean percentage increases over control LCGU at 0.3 and $1.0 \mathrm{mg} / \mathrm{kg}$ nicotine, respectively): cerebellar vermis $(66 \%$, $71 \%)$, presubiculum $(33 \%, 43 \%)$, subiculum $(36 \%, 31 \%)$, superior colliculus $(112 \%, 91 \%)$, red nucleus $(36 \%, 28 \%)$, ventral tegmental area $(45 \%, 36 \%)$, interpeduncular nucleus $(51 \%, 47 \%)$, dorsal lateral geniculate nucleus $(46 \%, 37 \%)$, substantia nigra pars compacta $(40 \%, 36 \%)$, anteroventral thalamic nucleus $(59 \%$, $78 \%)$, interanteromedial thalamic nucleus $(47 \%, 50 \%)$, median eminence $(161 \%, 96 \%)$, fasciculus retroflexus $(40 \%, 115 \%)$, mammillothalamic tract $(30 \%, 35 \%)$, and fornix $(39 \%, 42 \%)$. Some areas showed a significant enhancement of LCGU after $0.3 \mathrm{mg} / \mathrm{kg}$ nicotine, whereas in others the effect did not reach statistical significance after $1.0 \mathrm{mg} / \mathrm{kg}$ nicotine. These areas included the reticular nucleus of the medulla $(28 \%, 11 \%)$, the paramedian lobule $(35 \%, 18 \%)$, and nucleus of the spinal tract of the trigeminal nerve $(36 \%, 19 \%)$. In general, effects of 1.75 $\mathrm{mg} / \mathrm{kg}$ nicotine were like those of $1.0 \mathrm{mg} / \mathrm{kg}$ (London et al., 1985a).

Some areas showed greater LCGU effects after $1.0 \mathrm{mg} / \mathrm{kg}$ than $0.3 \mathrm{mg} / \mathrm{kg}$ nicotine. In the medial habenula and the nucleus of the solitary tract, significant effects were reached only at $1.0 \mathrm{mg}$ $\mathrm{kg}(111 \%$ and $29 \%$ over control, respectively). In the fasciculus retroflexus, LCGU was significantly elevated at $0.3 \mathrm{mg} / \mathrm{kg}(40 \%)$, but the effect of $1.0 \mathrm{mg} / \mathrm{kg}$ was greater than that of $0.3 \mathrm{mg} / \mathrm{kg}$ (115\%).

In most areas assayed, the effect on LCGU dissipated by 15 min after nicotine treatment (Table 2). Only 2 regions showed a longer duration of the effect. One of them was the medial habenula, in which the effect was observed in rats given nicotine
$15 \mathrm{~min}$, but not 60 or $120 \mathrm{~min}$, before ${ }^{14} \mathrm{C}-\mathrm{DG}$, and the superior colliculus, in which the effect was sustained for $120 \mathrm{~min}$.

All regions assayed showed antagonism of nicotine-induced LCGU stimulation by prior treatment with mecamylamine (2.5 $\mathrm{mg} / \mathrm{kg}$, s.c.) (Table 3). Mecamylamine completely blocked the stimulation in all regions except the interpeduncular nucleus, where there was still an LCGU increase of about $30 \%$ over

Table 3. Effect of mecamylamine on nicotine's stimulation of local cerebral glucose utilization

\begin{tabular}{lrc} 
& $\begin{array}{l}\text { Saline } \\
\text { control } \\
(n=7)\end{array}$ & $\begin{array}{l}\text { Mecamyl- } \\
\text { amine+ } \\
\text { nicotine } \\
(n=4)\end{array}$ \\
\hline Cerebellar vermis, granular layer & $63 \pm 4.1$ & $64 \pm 3.4$ \\
Presubiculum & $100 \pm 7.2$ & $116 \pm 8.0$ \\
Superficial gray, superior colliculus & $78 \pm 5.2$ & $72 \pm 2.7$ \\
Ventral tegmental area & $66 \pm 4.2$ & $67 \pm 1.7$ \\
Interpeduncular n., central & $115 \pm 7.6$ & $151 \pm 11^{*}$ \\
Dorsal lateral geniculate $\mathrm{n}$. & $83 \pm 5.9$ & $87 \pm 4.0$ \\
Substantia nigra pars compacta & $72 \pm 4.6$ & $79 \pm 4.8$ \\
Medial habenular n. & $91 \pm 11$ & $94 \pm 9.5$ \\
Anteroventral thalamic n. & $117 \pm 7.2$ & $129 \pm 7.3$ \\
N. solitary tract & $59 \pm 2.2$ & $62 \pm 2.2$
\end{tabular}

Rats were given mecamylamine $(2.5 \mathrm{mg} / \mathrm{kg}) 20 \mathrm{~min}$ before $d, l$-nicotine bitartrate $(1.0 \mathrm{mg} / \mathrm{kg})$, which was injected $2 \mathrm{~min}$ before ${ }^{14} \mathrm{C}$-DG. The drugs were injected subcutaneously in $1 \mathrm{ml} / \mathrm{kg}$ body weight of $0.9 \% \mathrm{NaCl}$ (wt/vol). Control rats were given subcutaneous injections of the same volume of $0.9 \% \mathrm{NaCl} 2$ min before ${ }^{14} \mathrm{C}$-DG. For each brain region, at least 6 densitometric readings were taken from no less than 3 tissue sections. Drug dosages are expressed as the free base. The values for LCGU ( $\mu \mathrm{mol} / 100 \mathrm{gm}$ tissuc/min) arc cxpressed as mcans \pm SEM for the number of rats indicated in parentheses. Asterisk indicates significant difference from control ( $p \leq 0.05$ by Student's $t$ test). 

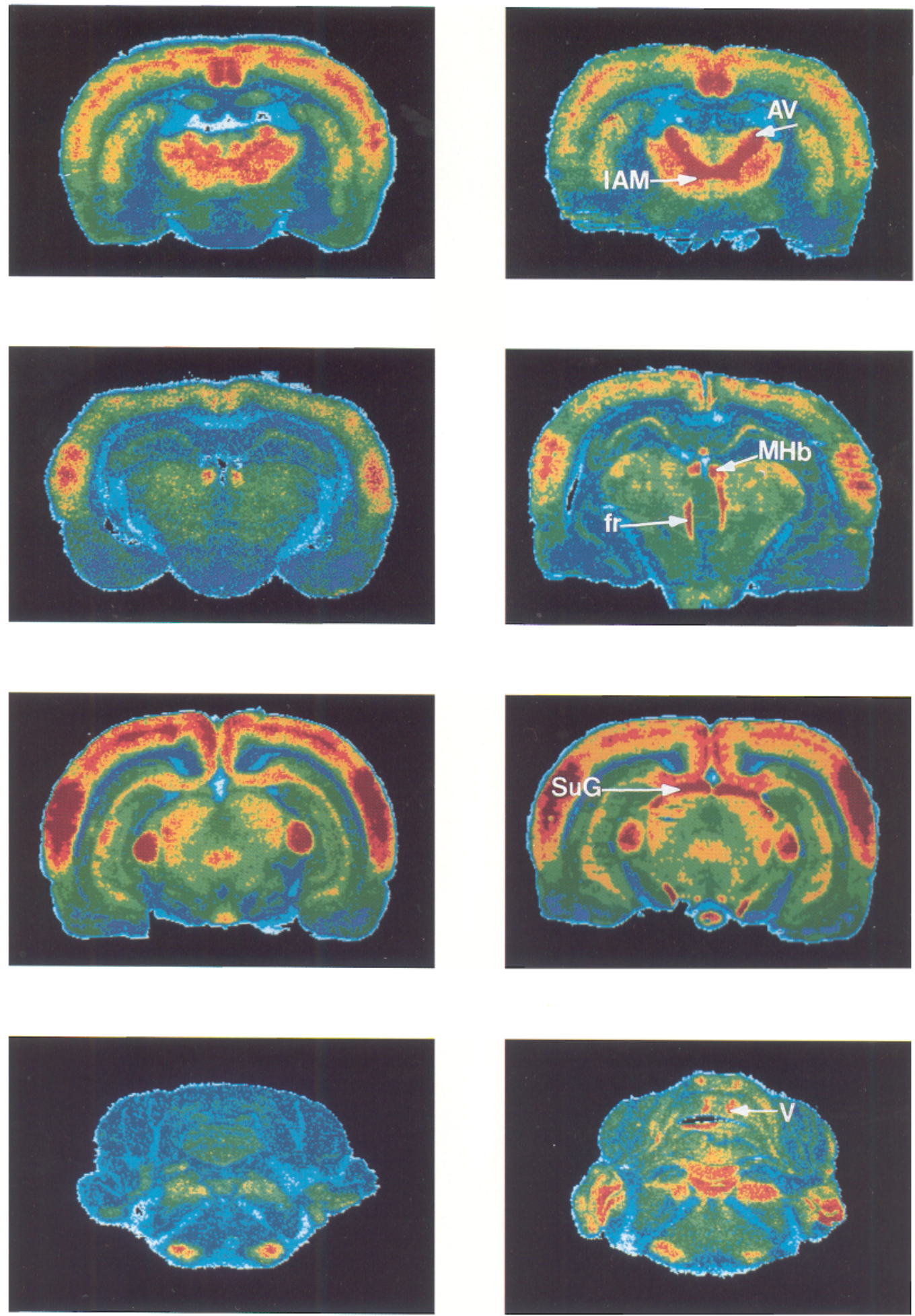

Figure 3. Quantitative color-coded transforms of autoradiographs showing the rates of glucose utilization in sections from different brain levels in control rats (left) and rats treated with 0.3 or $1.0 \mathrm{mg} / \mathrm{kg}$ nicotine, s.c., $2 \mathrm{~min}$ before ${ }^{14} \mathrm{C}-\mathrm{DG}$ (right). Higher rates of glucose utilization are indicated by warmer colors (red $>$ orange $>$ yellow $>$ green $>$ blue), with the highest rates $(\geq 176 \mu \mathrm{mol} / 100$ gm tissue/min) depicted as red. Nicotine stimulated glucose utilization in the anteroventral $(A V)$ and interanteromedial $(I A M)$ thalamic nuclei, medial habenular nucleus $(M H b)$, fasciculus retroflexus $(f r)$, and superficial gray layer of the superior colliculus $(S u G)$. Nicotine-treated rats also showed hypermetabolic spots, arranged vertically in the cerebellar vermis $(V)$. Scale bar, $0.5 \mathrm{~cm}$. 
control (as compared with $47 \%$ in rats treated with nicotine but no mecamylamine, Table 1). Preliminary studies showed that hexamethonium $(4.0 \mathrm{mg} / \mathrm{kg}$, i.p., $10 \mathrm{~min}$ pretreatment $)$ did not block the effects of nicotine $\left(1.75 \mathrm{mg} / \mathrm{kg} 2 \mathrm{~min}\right.$ before ${ }^{14} \mathrm{C}-\mathrm{DG}$, data not shown).

\section{Discussion}

The effects of nicotine on LCGU generally follow the localization of ${ }^{3} \mathrm{H}$-nicotine binding in the rat brain (Clarke et al., 1984, 1985; London et al., 1985a, b). High densities of ${ }^{3} \mathrm{H}$-nicotine binding sites have been measured in the medial habenula, interpeduncular nucleus, and superior colliculus (London et al., 1985b), which show marked LCGU stimulation by nicotine. Dense binding also occurs in thalamic nuclei, the dorsal lateral geniculate nucleus, substantia nigra pars compacta, ventral tegmental area, presubiculum, and subiculum (Clarke et al., 1985; London et al., 1985b), which show nicotine-induced LCGU activation. The very sparse labeling in Ammon's horn and the dentate gyrus with ${ }^{3} \mathrm{H}-\mathrm{ACh}$ and ${ }^{3} \mathrm{H}$-nicotine (Schwartz et al., 1982; London et al., 1985b) suggests that the lack of LCGU responses to nicotine in these areas is not coincidental. Areas with moderate binding, such as the cerebellar vermis and paramedian lobule, also show LCGU responses to nicotine. Increased LCGU in areas rich in ${ }^{3} \mathrm{H}$-nicotine binding sites indicates that the sites are true receptors.

There are some discrepancies between the distributions of LCGU responses to nicotine and ${ }^{3} \mathrm{H}$-nicotine binding. Some areas with moderate binding, such as the caudate-putamen and parts of the neocortex, show no LCGU increases. Such cases may reflect dilution of metabolic effects in brain areas where LCGU reflects Inetabolism in heterogeneous cell populations. Alternatively, some specific ${ }^{3} \mathrm{H}$-nicotine binding sites may not be true receptors. LCGU stimulation in a few areas that are not enriched in ${ }^{3} \mathrm{H}$-nicotine binding sites (red nucleus, median eminence) could be indirect, as LCGU effects may be propagated via afferents to areas remote from initial receptor interactions.

Blockade of nicotine effects on LCGU by mecamylamine but not hexamethonium agrees with a central agonistic action of nicotine. Many reports have shown a blockade of behavioral effects of nicotine by mecamylamine but not hexamethonium, which does not readily cross the blood-brain barrier (e.g., Schechter and Rosecrans, 1971; Clarke and Kumar, 1983; review, Stolerman, 1986).

The present dose-response and time-course data allow correlations with previous behavioral findings. Nicotine dosages that affected LCGU were comparable to those which influenced behavior in rats. Stimulation in most areas was produced by $0.3 \mathrm{mg} / \mathrm{kg}$ nicotine, a dose similar to those used in training rats to discriminate nicotine (Overton, 1979). An effect on LCGU in most areas was not measurable after $15 \mathrm{~min}$, although $1 \mathrm{mg} /$ $\mathrm{kg}$ nicotine s.c. produced nearly maximal drug concentrations in the rat brain at that time (Tripathi et al., 1982). The discrepancy may reflect tachyphylaxis, as tachyphylaxis to nicotine-induced antinociception occurred in rats within $10 \mathrm{~min}$ (Tripathi et al., 1982). The superior colliculus and the medial habenula appeared to be less susceptible to such tachyphylaxis than other brain areas assayed.

The use of metabolic mapping to visualize activated circuits in the brain, including fiber pathways, is demonstrated by the nicotine-induced stimulation of the habenulo-interpeduncular system. The medial habenula projects to the interpeduncular nucleus via the fasciculus retroflexus (Contestabile and Flumerfelt, 1981; also see review by Faull and Mehler, 1985), mainly by cholinergic inputs (see review by Paxinos and Butcher, 1985). The habenulo-interpeduncular system contains surprisingly fewer muscarinic cholinergic receptors (Rotter et al., 1979) than nicotinic ones (Clarke et al., 1984; London et al., 1985b), suggesting that the cholinergic transmission here may be largely nicotinic.

The Papez circuit (Papez, 1937), which links the hippocampal formation, anterior thalamus, mammillary bodies, and medial cortex (Nauta, 1956; Swanson and Cowan, 1975, 1977; Wyss et al., 1979) shows nicotine-induced stimulation. Nicotine stimulates LCGU in the anteroventral thalamic nucleus, in the subicular area of the hippocampal formation, and (nonsignificantly) in the medial mammillary nucleus. LCGU also is markedly enhanced in the fornix and mammillothalamic tract. Lesions of Papez circuit components and their associated fiber tracts disrupt performance in memory testing paradigms (Krieckhaus, 1964, 1967; Van Buren and Borke, 1972; Hirsh et al., 1979; Moss et al., 1981). Furthermore, oxotremorine, a muscarinic agonist that enhances performance on such tests, stimulates LCGU in the Papez circuit (Dam and London, 1984). It therefore has been suggested that cholinergic drug effects in the Papez circuit might alter learning, memory, or emotional, and/or motivational factors which influence performance in learning and memory testing paradigms (Dam and London, 1984). As nicotine facilitates performance in learning and memory tests (e.g., Bovet et al., 1969; Larson and Silvette, 1975; Warburton et al., 1986), it seems feasible that effects of nicotine on cognitive performance may reflect an action in the Papez circuit.

Nicotine fulfills the criteria for a reinforcing drug stimulus in laboratory animals and humans (Dougherty et al., 1981; Henningfield and Goldberg, 1983); however, the mechanism of reinforcement is unknown. Nicotine stimulates LCGU in the ventral tegmental area and the habenular complex, which have been implicated in drug- and stimulation-induced reward, respectively (Wise, 1980; Nakajima, 1984). Our findings, therefore, suggest a possible involvement of these areas in the reinforcing properties of nicotine.

Mice treated with nicotine show tremors, restlessness, increased motor activity, and the Straub-tail response; higher doses produce convulsions (Saxena et al., 1976). The motor effects of nicotine in the present study were consistent with previous findings (Larson et al., 1961; Saxena et al., 1976) and were associated with LCGU increases in several brain areas involved in motor function, such as the cerebellar vermis, paramedian lobule, red nucleus, and substantia nigra pars compacta, but other forebrain or cortical motor areas were unaffected. Nicotine-induced stimulation of LCGU in the cerebellar vermis and the paramedial lobule may relate to effects of nicotine on motor coordination. Like nicotine, oxotremorine also produces tremor and increases LCGU in the cerebellar vermis, red nucleus, and substantia nigra pars compacta (Dow-Edwards et al., 1981), supporting a role of these areas in tremor.

Nicotine produces antinociception in laboratory animals apparently by a central action (Mansner, 1972; Phan et al., 1973). The stimulation of LCGU in the nucleus of the spinal tract of the trigeminal nerve (Sp5) may be relevant to the antinociceptive action of nicotine, as this brain area is a sensory nucleus (Tracey, 1985). In addition, the interpolar subnucleus of Sp5 projects to the cerebellar vermis (Watson and Switzer, 1978) and the superior colliculus (Huerta et al., 1983). Therefore, some 
of the LCGU stimulation by nicotine in the latter sites may reflect activity in afferents from $\mathrm{Sp} 5$.

Other physiological effects of nicotine include an inhibition of increased prolactin secretion during proestrous and in response to suckling in rats (Blake and Sawyer, 1972; Blake et al., 1973). Repeated nicotine treatment reduces catecholamine stores in the medial palisade zone of the median eminence, suggesting that nicotine lowers prolactin secretion via effects on catecholamine terminals in the median eminence (Fuxe et al., 1977). LCGU stimulation in the median eminence is consistent with this view and suggests that enhanced metabolic activity and catecholamine turnover in terminals of the median eminence are involved in the effects of nicotine on prolactin.

Nicotine elevates blood pressure and LCGIJ in the nucleus ambiguus and nucleus of the solitary tract. Although such parallel findings do not prove a causal relation, the connections of the nucleus of the solitary tract and the nucleus ambiguus (see review by Bystrzycka and Nail, 1985) indicate an involvement of these nuclei in the central integration of autonomic function (for review, see Kirchheim, 1976). The present effects of nicotine on blood pressure appear to have resulted at least in part from stimulation of the sympathoadrenal system ( $\mathrm{Su}, 1982)$. Supporting this view, both hexamethonium and mecamylamine effectively prevented nicotine-induced hypertension. Therefore, LCGU stimulation in the nucleus of the solitary tract and nucleus ambiguus could have been secondary to hypertension, reflecting a compensatory central mechanism to restore normotension (Gabriel and Scller, 1970). The LCGU cffect in the nucleus of the solitary tract could plausibly have been mediated by afferents either from the ventral medulla or directly from the baroreceptors (for review, see Palkovits and Zaborszky, 1977).

In summary, nicotine effects on LCGU generally parallel the distributions of specific nicotine binding sites in the rat brain, indicating that the sites are true receptors. LCGU effects are seen at dosages that are discriminated by the rat and produce behavioral and physiological effects. The present findings provide new information relevant to elucidating the brain areas that mediate some of the behavioral and physiological effects of nicotine.

\section{References}

Aceto, M. D., and B. R. Martin (1982) Central actions of nicotine. Med. Res. Rev. 2: 43-62.

Blake, C. A., and C. H. Sawyer (1972) Nicotine blocks the sucklinginduced rise in circulating prolactin in lactating rats. Science 177 . 619-621.

Blake, C. A., R. L. Norman, R. J. Scaramuzzi, and C.H. Sawyer (1973) Inhibition of the proestrous surge of prolactin in the rat by nicotine. Endocrinology 92: 1334-1338.

Bovet, D., F. Bovet-Nitti, and A. Oliverio (1969) Genetic aspects of learning and memory in mice. Science 163: 139-149.

Bystrzycka, E. K., and B. S. Nail (1985) Brain stem nuclei associated with respiratory, cardiovascular and other autonomic functions. In The Rat Nervous System, Vol. 2, G. Paxinos, ed., pp. 95-110, Academic, North Ryde, Australia.

Clarke, P. B. S., and R. Kumar (1983) The effects of nicotine on locomotor activity in non-tolerant and tolerant rats. Br. J. Pharmacol. 78: 329-337.

Clarke, P. B. S., C. B. Pert, and A. Pert (1984) Autoradiographic distribution of nicotine receptors in rat brain. Brain Res. 323: 390395.

Clarke, P. B. S., R. D. Schwartz, S. M. Paul, C. B. Pert, and A. Pert (1985) Nicotinic binding in rat brain: Autoradiographic comparison of $\left[{ }^{3} \mathrm{H}\right]$ acetylcholine, $\left[{ }^{3} \mathrm{H}\right]$ nicotine, and $\left[{ }^{125} \mathrm{I}\right]-\alpha$-bungarotoxin. J. Neurosci. 5: 1307-1315.

Contestabile, A., and B. A. Flumerfelt (1981) Afferent connections of the interpeduncular nucleus and the topographic organization of the habenulo-interpeduncular pathway: An HRP study in the rat. J. Comp. Neurol. 196: 253-270.

Dam, M., and E. D. London (1984) Glucose utilization in the Papez circuit: Effects of oxotremorine and scopolamine. Brain Res. 295. $137-144$.

Dougherty, J., D. Miller, G. Todd, and H. B. Kostenbauder (1981) Reinforcing and other behavioral effects of nicotine. Neurosci. Biobehav. Rev. 5: 487-495.

Dow-Edwards, D., M. Dam, J. M. Peterson, S. I. Rapoport, and E. D. London (1981) Effect of oxotremorine on local cerebral glucose utilization in motor system regions of the rat brain. Brain Res. 226 . 281-289.

Esposito, R. U., L. J. Porrino, T. F. Seeger, A. M. Crane, H. D. Everist and A. Pert (1984) Changes in local cerebral glucose utilization during rewarding brain stimulation. Proc. Natl. Acad. Sci. USA 81: 635-639.

Faull, R. L. M., and W. R. Mehler (1985) Thalamus. In The Rat Nervous System, Vol. 1, G. Paxinos, ed., pp. 129-168, Academic, North Ryde, Australia.

Fuxe, K., L. Agnati, P. Eneroth, J.-A. Gustafsson, T. Hökfelt, A. Löfström, B. Skett, and P. Skett (1977) The effect of nicotine on central catecholamine neurons and gonadotropin secretion. I. Studies in the male rat. Med. Biol. 55: 148-157.

Gabriel, M., and H. Seller (1970) Interaction of baroreceptor afferents from carotid sinus and aorta at the nucleus tractus solitarii. Pfluegers Arch. 318: 7-20.

Henningfield, J. E. (1984) Behavioral pharmacology of cigarette smoking. In Advances in Behavioral Pharmacology, Vol. 4, T. Thompson, P. B. Dews, and J. E. Barrett, eds., pp. 131-210, Academic, New York.

Henningfield, J. E., and S. R. Goldberg (1983) Nicotine as a reinforcer in human subjects and laboratory animals. Pharmacol. Biochem. Behav. 19: 989-992.

Hirsh, R., R. E. Davis, and L. Holt (1979) Fornix-thalamus fibers, motivational states and contextual retrieval. Exp. Neurol. 65: 373390.

Huerta, M. F., A. Frankfurter, and J. K. Harting (1983) Studies of the principal sensory and spinal trigeminal nuclei of the rat: Projections to the superior colliculus, inferior olive, and cerebellum. J. Comp. Neurol. 220: 147-167.

Ito, M., M. Kadekaro, and L. Sokoloff (1985) Effects of lateral habenular lesions on local cerebral glucose utilization in the rat. Brain Res. 337: 245-254.

Kirchheim, H. R. (1976) Systemic arterial baroreceptor reflexes. Physiol. Rev. 56: 100-176.

Krieckhaus, E. E. (1964) Decrements in avoidance behavior following mammillothalamic tractotomy in cats. J. Neurophysiol. 27: 753-767.

Krieckhaus, E. E. (1967) The mammillary bodies: Their function and anatomical connections. Acta Biol. Exp. 27: 319-337.

Larson, P. S., and H. Silvette (1968) Tobacco: Experimental and Clinical Studies, Suppl. I, Williams \& Wilkins, Baltimore.

Larson, P. S., and H. Silvette (1971) Tobacco: Experimental and Clinical Studies, Suppl. II, Williams \& Wilkins, Baltimore.

Larson, P. S., and H. Silvette (1975) Tobacco: Experimental and Clinical Studies, Suppl. III, Williams \& Wilkins, Baltimore.

Larson, P. S., H. B. Haag, and H. Silvette (1961) Tobacco: Experimental and Clinical Studies. A Comprehensive Account of World Literature, Williams \& Wilkins, Baltimore.

London, E. D., S. M. Nespor, M. Ohata, and S. I. Rapoport (1981) Local cerebral glucose utilization during development and aging of the Fischer-344 rat. J. Neurochem. 37: 217-221.

London, E. D., M. McKinney, M. Dam, A. Ellis, and J. T. Coyle (1984) Decreased cortical glucose utilization after ibotenate lesion of the rat ventromedial globus pallidus. J. Cereb. Blood Flow Metab. 4: 381390.

London, E. D., R. J. Connolly, M. Szikszay, and J. K. Wamsley (1985a) Distribution of cerebral metabolic effects of nicotine in the rat. Eur. J. Pharmacol. 110: 391-392.

London, E. D., S. B. Waller, and J. K. Wamsley (1985b) Autoradiographic localization of $\left[{ }^{3} \mathrm{H}\right]$ nicotine binding sites in the rat brain. Neurosci. Lett. 53: 179-184.

Mansner, R. (1972) Relation between some central effects of nicotine and its brain levels in the mouse. Annu. Med. Exp. Biol. Fenn. 50: 205-212. 
McCulloch, J. (1982) Mapping functional alterations in the CNS with $\left[{ }^{14} \mathrm{C}\right]$ deoxyglucose. In Handbook of Psychopharmacology, Vol. 15, L. L. Iversen, S. D. Iversen, and S. H. Snyder, eds., pp. 321-410, Plenum, New York.

Moss, M., H. Mahut, and S. Zola-Morgan (1981) Concurrent discrimination learning of monkeys after hippocampal, entorhinal, or fornix lesions. J. Neurosci. 1: 227-240.

Nakajima, S. (1984) Serotonergic mediation of habenular self-stimulation in the rat. Pharmacol. Biochem. Behav. 20:859-862.

Nauta, W. J. W. (1956) An experimental study of the fornix system in the rat. J. Comp. Neurol. 104: 247-271.

Ovcrton, D. A. (1979) Drug discrimination training with progrcssivcly lowered doses. Science 205: 720-721.

Palkovits, M., and L. Zaborszky (1977) Neuroanatomy of central cardiovascular control. Nucleus tractus solitarii: Afferent and efferent neuronal connections in relation to the baroreceptor reflex arc. Prog. Brain Res. 47: 9-34.

Papez, J. W. (1937) A proposed mechanism of emotion. Arch. Neurol. Psychiatry 38: 725-744.

Paxinos, G., and L. L. Butcher (1985) Organizational principles of the brain as revealed by choline acetyltransferase and acetylcholinesterase distribution and projections. In The Rat Nervous System, Vol. 1, G. Paxinos, ed., pp. 471-522, Academic, North Ryde, Australia.

Paxinos, G., and C. Watson (1982) The Rat Brain in Stereotaxic Coordinates, Academic, New York.

Phan, D. V., M. Doda, A. Bilc, and L. Gyorgy (1973) Antinociceptive activity of nicotine. Acta Physiol. Acad. Sci. Hung. 1: 85-93.

Rotter, A., N. J. M. Birdsall, A. S. Burgen, P. M. Field, E. D. Hulme, and G. Raisman (1979) Muscarinic receptors in the central nervous system of the rat. I. Technique for autoradiographic localization of the binding of $\left[{ }^{3} \mathrm{H}\right]$ propylbenzylcholine mustard and its distribution in the forebrain. Brain Res. Rev. 180: 141-165.

Saxena, R. C., K. S. Dixit, K. M. Dhasmana, and R. P. Kohli (1976) Effect of nicotine administration into the lateral cerebral ventricles of mice provides evidence for cholinergic mechanisms in the CNS. In Drugs and Central Synaptic Transmission, P. B. Bradley and B. N. Dhawan, eds., pp. 139-144, University Park Press, Baltimore.

Schechter, M. D., and J. A. Rosecrans (1971) CNS effects of nicotine as the discriminative stimulus for the rat in a T-maze. Life Sci. 10: $821-832$.
Schwartz, R. D., R. McGee, Jr., and K. J. Kellar (1982) Nicotinic cholinergic receptors labeled by $\left.{ }^{3} \mathrm{H}\right]$ acetylcholine in rat brain. Mol. Pharmacol. 22: 56-62.

Sokoloff, L. (1977) Relation between physiological function and energy metabolism in the central nervous system. J. Neurochem. 29: 13-26.

Sokoloff, L., M. Reivich, C. Kennedy, M. Des Rosiers, C. Patlak, K. Pettigrew, O. Sakurada, and M. Shinohara (1977) The $\left[{ }^{14} \mathrm{C}\right]$ deoxyglucose method for the measurement of local cerebral glucose utilization: Theory, procedure and normal values in the conscious and anesthetized albino rat. J. Neurochem. 28: 897-916.

Stolerman, I. P. (1986) Could nicotine antagonists be used in smoking ccssation? Br. J. Addiction 81: 47-53.

$\mathrm{Su}, \mathrm{C}$. (1982) Actions of nicotine and smoking on circulation. Pharmacol. Ther. 17: 129-141.

Swanson, L. W., and W. M. Cowan (1975) Hippocampo-hypothalamic connections: Origin in subicular cortex, not Ammon's horn. Science 189: 303-304.

Swanson, L. W., and W. M. Cowan (1977) An autoradiographic study of the organization of the efferent connections of the hippocampal formation in the rat. J. Comp. Neurol. 172: 49-84.

Tracey, D. J. (1985) Somatosensory system. In The Rat Nervous System, Vol. 2, G. Paxinos, ed., pp. 129-152, Academic, North Ryde, Australia.

Tripathi, H. L., B. R. Martin, and M. D. Aceto (1982) Nicotineinduced antinociception in rats and mice: Correlation with nicotine brain levels. J. Pharmacol. Exp. Ther. 221: 91-96.

Van Buren, J. M., and R. C. Borke (1972) The mesial temporal substratum of memory. Anatomical studies in three individuals. Brain 95: 599-632.

Warburton, D. M., K. Wesnes, K. Shergold, and M. James (1986) Facilitation of learning and state dependency with nicotine. Psychopharmacology 89: 55-59.

Watson, C. R. R., and R. C. Switzer (1978) Trigeminal projections to cerebellar tactile areas in the rat-origin mainly from $n$. interpolaris and n. principalis. Neurosci. Lett. 10:77-82.

Wise, R. A. (1980) Action of drugs of abuse on brain reward systems. Pharmacol. Biochem. Behav., Suppl. 1, 13:213-2.23.

Wyss, J. M., L. W. Swanson, and W. M. Cowan (1979) A study of subcortical afferents to the hippocampal formation in the rat. Neuroscience 4: $463-476$. 